\title{
Balance hídrico en plantaciones de Pinus radiata en el NO de la Patagonia argentina
}

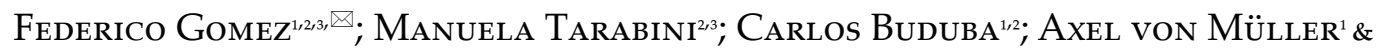 \\ Ludmila La ManNA ${ }^{2 / 3}$ \\ ${ }^{1}$ Instituto Nacional de Tecnología Agropecuaria (INTA), Estación Experimental Agroforestal Esquel. Chubut, Argentina. ${ }^{2}$ \\ Centro de Estudios Ambientales Integrados (CEAI). Facultad de Ingenierí, Universidad Nacional de la Patagonia San Juan \\ Bosco, Sede Esquel. Chubut, Argentina. ${ }^{3}$ Consejo Nacional de Investigaciones Científicas y Técnicas (CONICET). Argentina.
}

\begin{abstract}
Resumen. En la Región Andino Patagónica, el éxito de los sistemas silvopastoriles depende de la densidad de manejo forestal, que busca garantizar el mayor ingreso de agua al suelo para el forraje. El objetivo fue cuantificar y comparar los diferentes componentes del balance hídrico en una plantación de Pinus radiata con dos tipos de manejo: silvopastoril (SSP) y forestal tradicional (FT) (325 y 622 árboles/ha, respectivamente). Se midieron las variables que explican cómo se distribuye la precipitación que incide dentro de un bosque: precipitación directa, escurrimiento fustal, intercepción, humedad del suelo, percolación y evapotranspiración. Además, se determinó la cobertura del dosel arbóreo, el índice de área foliar (IAF) y la producción del componente forrajero. Con respecto al FT, el SSP presentó mayor precipitación directa $(84 \pm 1$ vs. $75 \pm 2 \%)$, menor escurrimiento fustal $(2 \pm 0.3$ vs. $3 \pm 0.27 \%)$, menor intercepción ( $14 \pm 1$ vs. $22 \pm 2 \%$ ), mayor humedad del suelo $(24.2 \pm 2.5$ vs. $20.9 \pm 2.1 \%$ ) y menor cobertura del dosel $(70.9 \pm 0.05 \mathrm{vs} .74 .89 \pm 0.34 \%)$. A su vez, la percolación $(0.33 \pm 0.12 \mathrm{vs.} 0.11 \pm 0.08 \%)$, la evapotranspiración (78.2 vs. $73.3 \%$ ), la producción de pastos $\left(230.76 \pm 97.57 \mathrm{vs} .66 .6 \pm 60.02 \mathrm{~kg} \mathrm{MS}\right.$ ha $\left.^{-1} . \mathrm{anno}^{-1}\right)$ y el IAF (1.28 \pm 0.04 vs. $1.31 \pm 0.02)$ fueron similares en ambos sistemas. Estos resultados evidencian que la densidad de manejo modificó sólo algunos componentes del balance hídrico. Sin embargo, el mayor ingreso de agua al SSP no se vio reflejado en una mayor producción de pastos. El manejo de la densidad en plantaciones es fundamental, en particular en SSP donde se deben potenciar los distintos componentes del sistema para usar de forma más eficiente el agua de lluvia.
\end{abstract}

[Palabras clave: cobertura del dosel, densidad de manejo, evapotranspiración, forraje]

Aвstract. Hydrological balance in Pinus radiata plantations from NW Argentinean Patagonia. In the Patagonia Andean Region, silvopastoral system success depends on forestry management, which seeks to guarantee highest water input into the soil to forage production. The aim of this study was to quantify and compare different components of the water balance in a Pinus radiata forest under two different management practices: silvopastoral and forest treatment (325 and 622 trees/ha, respectively). In order to explain incident precipitation distribution within the forest, different variables were measured: direct precipitation, stemflow, interception, soil moisture content, percolation and evapotranspiration. Additionally, canopy cover, leaf area index (LAI) and forage production was determined. Silvopastoral system, with respect to forest system, presented higher direct precipitation $(84 \pm 1$ vs. $75 \pm 2 \%)$, lower stemflow ( $2 \pm 0.3$ vs. $3 \pm 0.27 \%)$, lower interception $(14 \pm 1$ vs. $22 \pm 2 \%)$, higher soil moisture content $(24.2 \pm 2.5$ vs. $20.9 \pm 2.1 \%)$ and lower canopy cover $(70.9 \pm 0.05$ vs. $74.89 \pm 0.34 \%)$. In turn, percolation $(0.33 \pm 0.12$ vs. $0.11 \pm 0.08 \%)$, evapotranspiration $(78.2$ vs. $73.3 \%)$, pasture production $\left(230.76 \pm 97.57\right.$ vs. $66.60 \pm 60.02 \mathrm{~kg} \mathrm{MS}$ ha $\left.^{-1} . \mathrm{year}^{-1}\right)$ and LAI $(1.28 \pm 0.04 \mathrm{vs.} 1.31 \pm 0.02)$ were similar in both systems. These results show that management density modified only some components of water balance. However, adding more water the silvopastoral system was not reflected in an increase in pasture production. Density management is key, mainly in silvopastoral system, where different system components must be raised up for a more efficient water usage.

[Keywords: canopy cover, density management, evapotranspiration, forage]

Editor asociado: Esteban Jobbágy
Recibido: 18 de Junio de 2018

Aceptado: 5 de Abril de 2019 


\section{INTRODUCCIÓN}

En el ecotono de la Región Andino Patagónica, Argentina, existen alrededor de 800000 hectáreas con aptitud forestal para implantar coníferas exóticas de crecimiento rápido, como las del género Pinus (Loguercio and Deccechis 2006). A pesar de que las plantaciones pueden ser una buena alternativa como respuesta a problemas socio-económicos y ambientales (Mussat et al. 2013), actualmente sólo hay alrededor de 80000 hectáreas implantadas (Loguercio and Deccechis 2006). La principal causa de la subutilización del potencial forestal en la región es la cultura ganadera ya establecida como actividad económica por más de un siglo (Schlichter and Laclau 1998). La presión de pastoreo generó procesos graves de degradación de los suelos y pérdida de productividad (Andrade 2002). Una alternativa para diversificar la producción y mitigar el deterioro del suelo son los sistemas silvopastoriles de bosques implantados (Nair et al. 2009).

Los sistemas silvopastoriles permiten compatibilizar la producción de madera, forraje y ganado en un mismo campo y aprovechar las interacciones positivas de dichos componentes (Carranza and Ledesma 2009). Estos sistemas protegen al suelo de procesos erosivos porque mejoran su fertilidad, su estructura y su infiltración (Mahecha 2002). Además, puede contribuir al bienestar animal dado que los árboles actúan de reparo frente a las inclemencias climáticas (Pérez et al. 2008), lo que amortigua las temperaturas extremas (Bahamonde et al. 2009) y redistribuye el agua de las precipitaciones (Huber et al. 1985).

El cambio de cobertura arbórea con las intervenciones, desde una plantación densa con fines de producción de madera a un sistema silvopastoril (más abierto), provoca cambios en el ciclo del agua y en sus distintos componentes. Esto se debe a que la cantidad de agua que ingresa al suelo depende de la especie arbórea, del tipo de manejo y de las condiciones climáticas del sitio (Oyarzún et al. 1985; Crockford and Richardson 2000). En general, la precipitación es uno de los mayores aportes de agua para los sistemas boscosos (Barradas 1983), y en particular para las plantaciones de coníferas exóticas en la Región Andino Patagónica, donde las lluvias se concentran en otoño-invierno, desacopladas con respecto al período de crecimiento de las plantas (Schlichter and Laclau 1998).
El aporte efectivo de agua al suelo (precipitación efectiva) está dado por la precipitación directa, que es aquella que atraviesa el follaje y llega directamente al suelo (Huber and Oyarzún 1983), y el escurrimiento fustal, que es el agua que recorre los troncos una vez saturados y desciende hacia la base (Branson et al. 1981). El escurrimiento fustal se relaciona con la densidad de manejo (Oyarzún et al. 1985), el área proyectada por la copa y la forma de las ramas (Ford and Deans 1978). Por lo general, el aporte de agua por precipitación directa es más importante que el que canaliza los troncos de los árboles (Santa et al. 1989). No obstante, el agua que ingresa por el fuste entra rápidamente en contacto con las raíces y penetra profundamente en el perfil (Kimmins 1997); es importante para los árboles como autoabastecimiento de agua durante la época seca (Huber and Oyarzún 1983).

El agua que retienen las hojas, las ramas y los troncos de los árboles vuelve a la atmósfera por evaporación. Este proceso se denomina intercepción. El agua interceptada no alcanza la superficie del suelo y se pierde sin ingresar al sistema (Huber et al. 1998). La intercepción depende de la densidad del bosque y alcanza valores mayores en bosques con doseles cerrados que en abiertos (Ford and Deans 1978). También depende de las características foliares de la especie (Gyenge et al. 2009; Licata et al. 2011). Otros elementos principales en el balance hídrico son la escorrentía superficial y la percolación. El primero es de gran importancia en ambientes con pendientes fuertes y poca cobertura vegetal (Zuazo et al. 2002). Mientras tanto, la percolación es la proporción de agua de lluvia que ingresa al suelo, y resulta de la diferencia entre el agua que queda retenida y la que sale por infiltración (Jaramillo and Cháves 1999).

La evapotranspiración es otra componente clave en el ciclo hidrológico para los sistemas forestales, y en especial para los silvopastoriles, debido a las necesidades hídricas diferenciales de los árboles y de las pasturas (Jobbágy et al. 2006). Desagregar los componentes de la precipitación y el uso diferencial por parte de los diferentes estratos de los sistemas silvopastoriles representa una gran oportunidad para mejorar su eficiencia y su productividad. Asimismo, el agua que transpiran los componentes vegetales de los sistemas se traduce en productividad primaria (Jobbágy et al. 2006) herbácea o forestal, que depende de factores climáticos, del tipo de 
cobertura y del tipo de manejo (Allen et al. 2006), y es el objetivo a manejar con las intervenciones silvícolas. En este contexto, en el que las precipitaciones regionales más abundantes se distribuyen en los meses de otoño-invierno (abril-septiembre) - es decir, en la etapa en la que las temperaturas bajas limitan el crecimiento vegetal-, los sistemas silvopastoriles pueden ser una alternativa excelente para optimizar el escaso recurso hídrico y, además, para compatibilizar la producción de forraje y ganadera.

El balance hidrológico en sistemas silvopastoriles es clave ya que cambios en la densidad arbórea a través de tratamientos silvícolas (raleos), afectan de manera directa a la distribución de las precipitaciones, a las variaciones en el contenido de humedad del suelo y a los valores de evapotranspiración (Huber et al. 1985). En la Patagonia argentina, los escasos estudios sobre balance hídrico en plantaciones tienen un enfoque ecofisiológico. Dichos estudios demostraron que las plantaciones de Pinus ponderosa (Douglas ex Lawson) con baja densidad arbórea no impactan negativamente sobre los recursos hídricos en sitios xéricos (Gyenge et al. 2011). Por otro lado, en la Patagonia chilena, la cantidad de estudios sobre los procesos hidrológicos es mayor, y demuestran que las plantaciones de Pinus radiata (D. Don), en comparación con sitios abiertos, modifican los componentes del balance hidrológico. En particular, Pinus radiata posee un mayor consumo de agua por evapotranspiración, en comparación con sitios dominados por arbustos y pastos (Huber et al. 2007).

El objetivo general del presente trabajo es cuantificar y comparar los diferentes componentes del balance hídrico (i.e., precipitación directa, escurrimiento fustal, intercepción, contenido de humedad del suelo, percolación y evapotranspiración), en un bosque de Pinus radiata ubicado en la Región Andino Patagónica argentina, con dos tipos de manejo: sistema silvopastoril y sistema forestal tradicional. A su vez, se busca estimar la producción de los pastos para cada sistema. Para abordar este trabajo se plantea como hipótesis que el sistema silvopastoril, al permitir que llegue más agua de lluvia al suelo, resulta más ventajoso que un sistema forestal tradicional y alcanza una evapotranspiración mayor que se traduce en una productividad forrajera más elevada.

\section{MATERIALES y MÉTODOS}

\section{Área de estudio}

El estudio se llevó a cabo en la Unidad Demostrativa Silvopastoril del Campo Experimental Agroforestal INTA-Trevelin, provincia del Chubut, aproximadamente en $43^{\circ} 07^{\prime} 18.40^{\prime \prime} \mathrm{S}$ y $71^{\circ} 32^{\prime} 56.32^{\prime \prime} \mathrm{O}$. El clima es del tipo mediterráneo, con precipitaciones abundantes entre los meses de abril y septiembre (más de 70\% de la precipitación total), con $1000 \mathrm{~mm}$ de precipitación media anual, como se observa en el climograma realizado para el período 1970-2009 (Figura 1). Los suelos, derivados de cenizas volcánicas, corresponden al gran grupo Udands ( $\mathrm{La}$ Manna et al. 2008).

El sitio de estudio correspondió a un bosque de Pinus radiata de regeneración natural post incendio, de 27 años de edad. En parte de la superficie ( 3.5 ha) se realizó un raleo y poda en invierno de 2012 a fin de adecuar la plantación a un manejo silvopastoril. En un área contigua (2 ha) se adecuó la densidad en

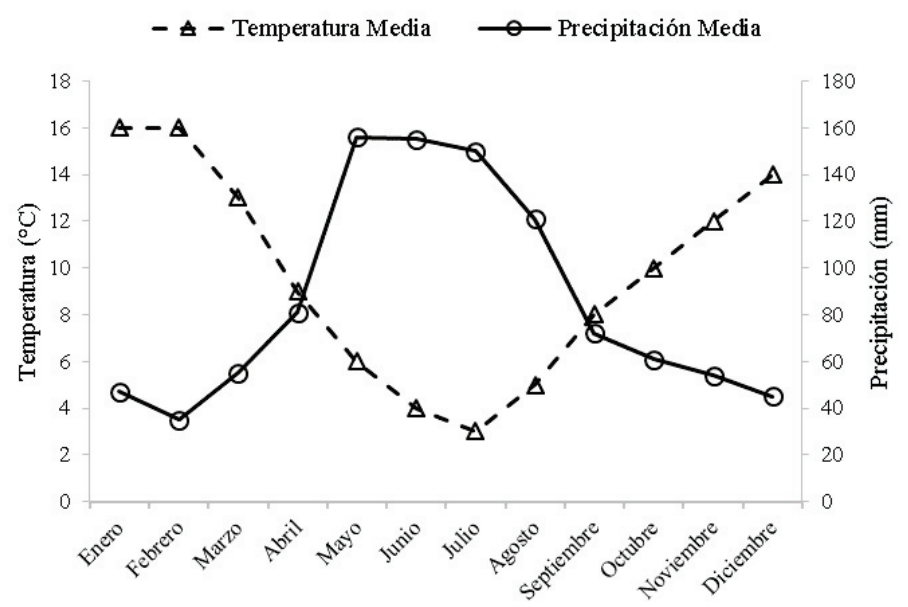

Figura 1. Climograma del sitio de estudio.

Figure 1. Study site climograph. 
otoño de 2014 para continuar con un manejo forestal tradicional. En cada sector (manejo silvopastoril vs. forestal tradicional) se instaló una parcela dasométrica circular de $706 \mathrm{~m}^{2}$. La densidad de árboles al momento de realizar el estudio en cada sector fue de 325 y 622 árboles ha $^{-1}$, respectivamente. Además, se estimó la cobertura del dosel y el índice de área foliar (IAF) a partir de 20 fotografías hemisféricas recolectadas en cada parcela: silvopastoril y forestal tradicional. Las imágenes se tomaron con una cámara Nikon Coolpix 5400 y se procesaron posteriormente con el programa Gap Light Analyzer v.2.0 (GLA) a fin de determinar la cobertura del dosel y el IAF para cada tratamiento, siguiendo la metodología que sugirieron Martínez Pastur et al. (2015).

\section{Precipitación}

Se registró la precipitación incidente $\left(\mathrm{P}_{\mathrm{i}}\right)$ con un pluviómetro ubicado a cielo abierto, a 50 $\mathrm{m}$ de la plantación. Dentro de cada parcela, abarcando la heterogeneidad del sistema, se midió la precipitación directa $\left(\mathrm{P}_{\mathrm{d}}\right)$ con una canaleta de $14.25 \mathrm{~m}$ de largo y $0.10 \mathrm{~m}$ de ancho $\left(1.4 \mathrm{~m}^{2}\right)$, que almacenó el agua en un bidón de 100 L, siguiendo la metodología sugerida por Huber y Trecamán (2004). El uso de una canaleta en lugar de pluviómetros puntuales es recomendable en este tipo de estudios, a fin de captar mejor la variabilidad de situaciones de cobertura dentro de un rodal. La canaleta se instaló a $1 \mathrm{~m}$ de altura, con una pendiente que aseguró un escurrimiento rápido hacia el bidón, evitando las pérdidas por evaporación (Buduba 2006); luego, los valores registrados se llevaron a hectárea. Para medir el escurrimiento fustal $\left(E_{f}\right)$ se seleccionaron 5 árboles dominantes en cada parcela, siguiendo el criterio sugerido por Buduba (2006). En cada uno de ellos se instaló un anillo helicoidal de aluminio que encausó el agua que bajaba por el tronco hacia un bidón de 50 L. Para determinar el escurrimiento fustal por hectárea se promediaron los valores medidos y se multiplicó por la densidad de árboles de cada parcela. La intercepción (I) se calculó por medio de la ecuación [1] (Huber and Oyarzún 1983, 1984); los valores se referenciaron a hectárea.

$$
\mathrm{I}=\mathrm{P}_{\mathrm{i}}-\mathrm{P}_{\mathrm{d}}-\mathrm{E}_{\mathrm{f}}
$$

donde

$\mathrm{I}$ = intercepción $(\mathrm{mm})$

$\mathrm{P}_{\mathrm{i}}=$ precipitación incidente $(\mathrm{mm})$
$\mathrm{P}_{\mathrm{d}}=$ precipitación directa $(\mathrm{mm})$

$\mathrm{E}_{\mathrm{f}}=$ escurrimiento fustal ( $\mathrm{mm}$ )

Las determinaciones se realizaron en 46 eventos de lluvia entre septiembre de 2014 y diciembre de 2015.

\section{Contenido de humedad del suelo}

Durante un año, desde septiembre de 2014 hasta agosto de 2015, se midió periódicamente la humedad del suelo. Para determinar la humedad gravimétrica en cada parcela, mensualmente se tomaron, con un barreno, tres muestras de suelo a $50 \mathrm{~cm}$ de profundidad; la profundidad $(40-60 \mathrm{~cm})$ corresponde a la sección de control de humedad para este tipo de suelos, de acuerdo con el Soil Survey Staff (2010). Además, para determinar las constantes hídricas del suelo también se tomaron 6 muestras en cada parcela. La capacidad de campo (CC; $0.01 \mathrm{MPa}$ ) y el punto de marchitez permanente (PMP; $1.5 \mathrm{MPa}$ ) se determinaron mediante olla y membrana de presión (López Ritas and López Melida 1990).

\section{Percolación}

La percolación fue estimada a partir de la diferencia entre el contenido de humedad a los $50 \mathrm{~cm}$ para cada tratamiento y la capacidad de campo medida, ecuación [2] (Huber and López 1993).

Per $=\mathrm{CH}-\mathrm{CC}$

donde

Per $=$ percolación $(\%)$

$\mathrm{CH}=$ contenido de humedad a los $50 \mathrm{~cm}(\%)$

$\mathrm{CC}=$ capacidad de campo $(\%)$

\section{Evapotranspiración}

En el período en que se midió la humedad del suelo, se estimó la evapotranspiración para ambos tipos de sistemas: silvopastoril y forestal tradicional a partir de la ecuación [3] (modificada a partir de Huber et al. 2007). En este análisis no se consideró el efecto de la escorrentía superficial debido a que la pendiente es menor a $2 \% \mathrm{y}$, por ende, la escorrentía es despreciable (Huber and Trecaman 2004).

$\mathrm{EVT}=\mathrm{P}_{\mathrm{i}}-(\mathrm{I}+\Delta \mathrm{W}+$ Per $)$

donde 
EVT = evapotranspiración $(\mathrm{mm})$

$\mathrm{P}_{\mathrm{i}}=$ precipitación incidente $(\mathrm{mm})$

$\mathrm{I}=$ intercepción $(\mathrm{mm})$

$\Delta \mathrm{W}=$ variación del contenido de humedad en el suelo ( $\mathrm{mm})$

Per $=$ percolación $(\mathrm{mm})$

Dado que en las parcelas de estudio no se midió la totalidad de los eventos de lluvia, para la estimación de EVT se consideraron todos los eventos de lluvia registrados con la estación meteorológica del INTA, ubicada a pocos metros del sector de estudio. Para aquellos eventos en que no se midió la intercepción, se la estimó a partir de la precipitación incidente, considerando la ecuación de regresión obtenida en este estudio (ver sección Resultados, Figura 5). A partir de los resultados de las distintas variables consideradas (precipitación incidente, precipitación directa, escurrimiento fustal, intercepción, contenido de humedad del suelo, percolación y evapotranspiración) se esbozó el balance hidrológico en los dos sistemas (silvopastoril y forestal tradicional).

\section{Producción de los pastos}

Durante el período de abril 2014 a marzo de 2015 se estimó la productividad primaria neta aérea del componente forrajero para los dos sistemas (silvopastoril y forestal tradicional) a partir de jaulas de corte. Se instalaron cuatro jaulas de hierro (con forma piramidal) de 2.25 $\mathrm{m}^{2}$ en cada uno de los tratamientos, y se realizó un sólo corte de la totalidad del material verde de $1 \mathrm{~m}^{2}$, en marzo de 2015 (evitando efecto borde de la jaula). El material cosechado fue puesto en estufa durante 72 horas hasta obtener peso constante, a fin de conseguir los kilogramos de materia seca por hectárea.

\section{Análisis de datos}

Las diferencias de las distintas variables (precipitación directa, escurrimiento fustal, intercepción, humedad del suelo, percolación, evapotranspiración, cobertura del dosel e
IAF) entre el sistema silvopastoril y forestal tradicional fueron analizadas mediante prueba de t para muestras apareadas. Mientras tanto, la producción de pastos se analizó por una prueba de t. La variación de la precipitación directa, el escurrimiento y la intercepción en función de la precipitación total fue analizada, para cada sistema, mediante regresiones lineales y no lineales. Las pendientes de las curvas de regresión lineal fueron comparadas entre ambos sistemas (silvopastoril vs. forestal tradicional) de acuerdo con la superposición o no de los intervalos de confianza. Se utilizó el software InfoStat $\circledR$.

El análisis de datos se realizó considerando como repeticiones los distintos eventos de lluvia. Como hay una única parcela por tratamiento, se trata de un muestreo sin repeticiones. No obstante, este tipo de análisis estadístico se considera adecuado para estudios de caso; por esta razón, nuestros resultados están acotados a la situación analizada, sin poder extrapolarlos (Hargrove and Pickering 1992; Huber and Trecamán 2004; Alinari et al. 2015).

\section{Resultados}

\section{Características de las parcelas}

Las parcelas presentaron grandes diferencias en densidad arbórea. Sin embargo, el DAP y la altura resultaron similares para ambos tratamientos (Tabla 1). La cobertura arbórea mostró diferencias significativas $(t=-6.43$; $P<0.0001$ ) (Figura 2), mientras que, el índice de área foliar fue similar para ambos tratamientos $(\mathrm{t}=-1.1 ; P=0.28)$.

\section{Precipitación}

En el período comprendido entre el 3/9/2014 y el 31/12/2015 se registraron 46 eventos de lluvia incidente en ambas parcelas (497.2 mm de lluvia medidos), lo cual representó sólo una parte de la precipitación total registrada en dicho período (1203 $\mathrm{mm}$ de lluvia). La precipitación directa media fue $84 \pm 1 \%$ de la lluvia incidente en la parcela silvopastoril y

Tabla 1. Características dasométricas y cobertura del dosel para los sistemas silvopastoril y forestal tradicional. Valores medios \pm EE.

Table 1. Dasometric characteristics and canopy cover for the silvopastoral and forest systems. Mean values \pm SE.

\begin{tabular}{lccccc}
\hline \multicolumn{1}{c}{ Sistema } & $\begin{array}{c}\text { DAP } \\
(\mathrm{cm})\end{array}$ & $\begin{array}{c}\text { Altura } \\
(\mathrm{m})\end{array}$ & $\begin{array}{c}\text { Densidad } \\
\text { (árboles/ha) }\end{array}$ & $\begin{array}{c}\text { Cobertura } \\
(\%)\end{array}$ \\
\hline Silvopastoril & $25.80 \pm 0.83$ & $17.4 \pm 0.48$ & 325 & $70.9 \pm 0.55$ & $1.28 \pm 0.04$ \\
Forestal tradicional & $23.35 \pm 0.65$ & $17.48 \pm 0.63$ & 622 & $74.89 \pm 0.34$ & $1.31 \pm 0.02$ \\
\hline
\end{tabular}



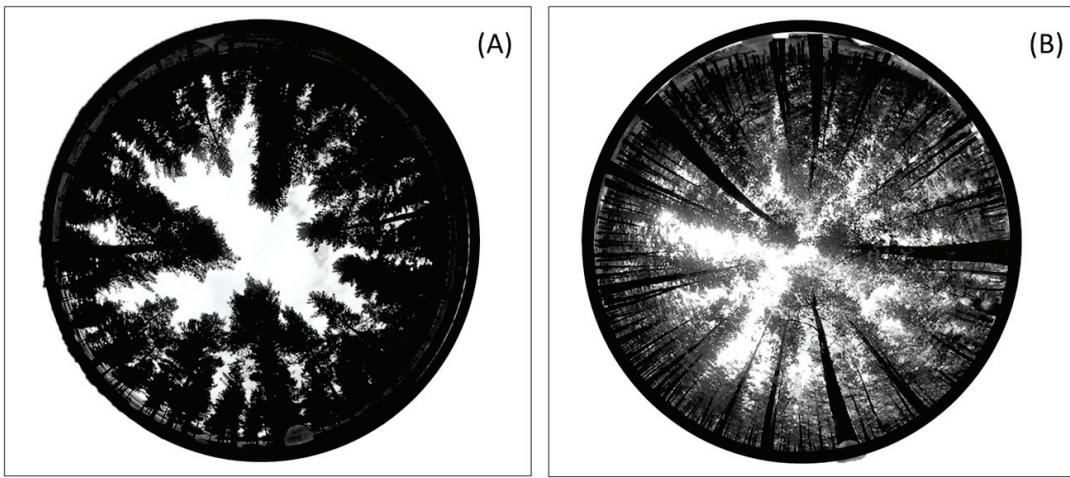

(B)

Figura 2. Fotografías hemisféricas de la cobertura del dosel para el sistema silvopastoril (A) y para el sistema forestal tradicional (B).

Figure 2. Hemispheric photographs of canopy cover for silvopastoral system (A) and forest system (B).

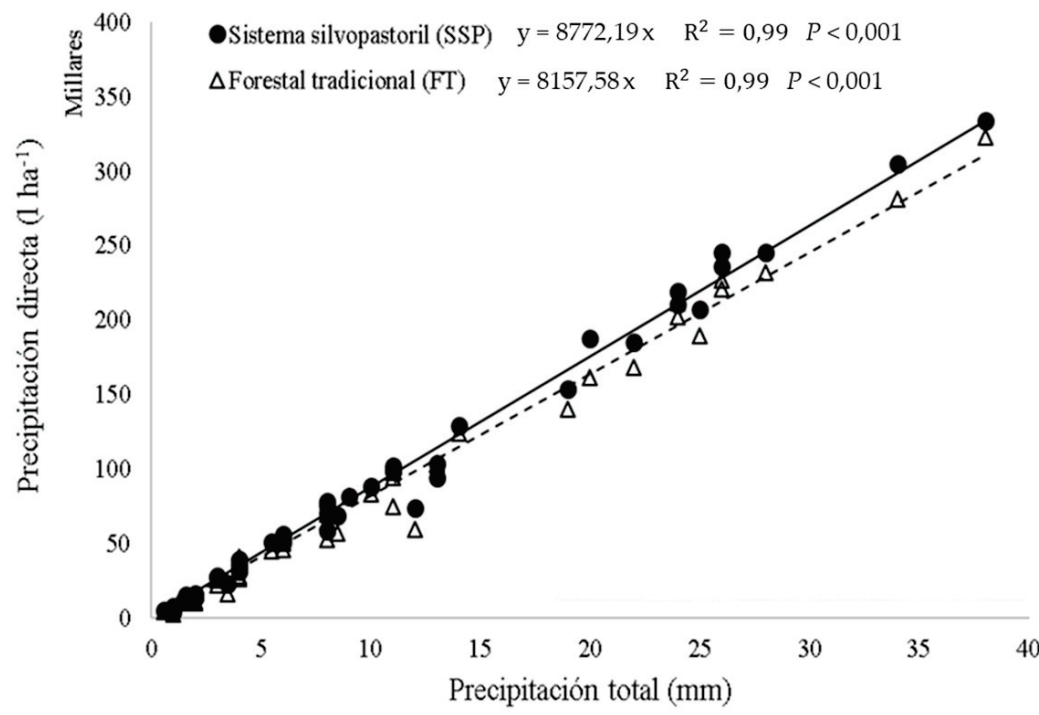

Figura 3. Precipitación directa registrada en el manejo silvopastoril (-) y en el forestal tradicional (---).

Figure 3. Throughfall in silvopastoral system (-) and forest system (---).

$75 \pm 2 \%$ en la forestal tradicional. El ingreso de agua fue significativamente mayor $(\mathrm{t}=6.59 ; P<0.001)$ en el sistema silvopastoril. La precipitación directa varió en relación a la precipitación total (Figura 3). Con eventos de precipitación mayores a $15 \mathrm{~mm}$, las diferencias entre las parcelas fueron más marcadas. La pendiente de la curva de regresión en el sistema silvopastoril (intervalo de confianza [8602.9 - 8941.5]) fue significativamente mayor que en el manejo forestal tradicional (intervalo de confianza [7991.6 - 8383.5]). Las pendientes de las curvas pueden ser interpretadas como un indicador de la eficiencia o la velocidad con que el agua llega al suelo, dependiendo de las condiciones ambientales de la evaluación.

El escurrimiento fustal evidenció también diferencias significativas $(\mathrm{t}=-4.35 ; P<0.01)$, con un valor medio de $2 \pm 0.3 \%$ de la lluvia incidente en la parcela silvopastoril y de $3 \pm 0.27 \%$ en la forestal tradicional. Con lluvias menores a $2 \mathrm{~mm}$, el agua que alcanzó el suelo moviéndose a través del tronco fue casi nula en ambas coberturas. Con precipitaciones mayores a $2 \mathrm{~mm}$, el escurrimiento fustal se incrementó en ambos manejos, con mayor aumento en la parcela forestal tradicional (Figura 4). A medida que las precipitaciones fueron más intensas, las diferencias entre ambos manejos fueron más marcadas. Los intervalos de confianza de las pendientes de las curvas de regresión fueron [309.5 - 373.3] y [392.8 - 465.4] para el manejo silvopastoril y manejo forestal tradicional, respectivamente; de esto se infiere que las pendientes difirieron significativamente entre ambos tratamientos.

El agua interceptada por el dosel fue siempre menor con el manejo silvopastoril (Figura 5). La intercepción media fue $14 \pm 1 \%$ para el manejo silvopastoril y $22 \pm 2 \%$ para la parcela forestal tradicional, presentando diferencias significativas $(\mathrm{t}=-6.17 ; P<0.001)$. Para ambos tratamientos, el porcentaje de intercepción fue mayor en lluvias de poca magnitud. A medida que las precipitaciones fueron más intensas, el porcentaje de intercepción disminuyó, tendiendo a un valor constante, pues el dosel 

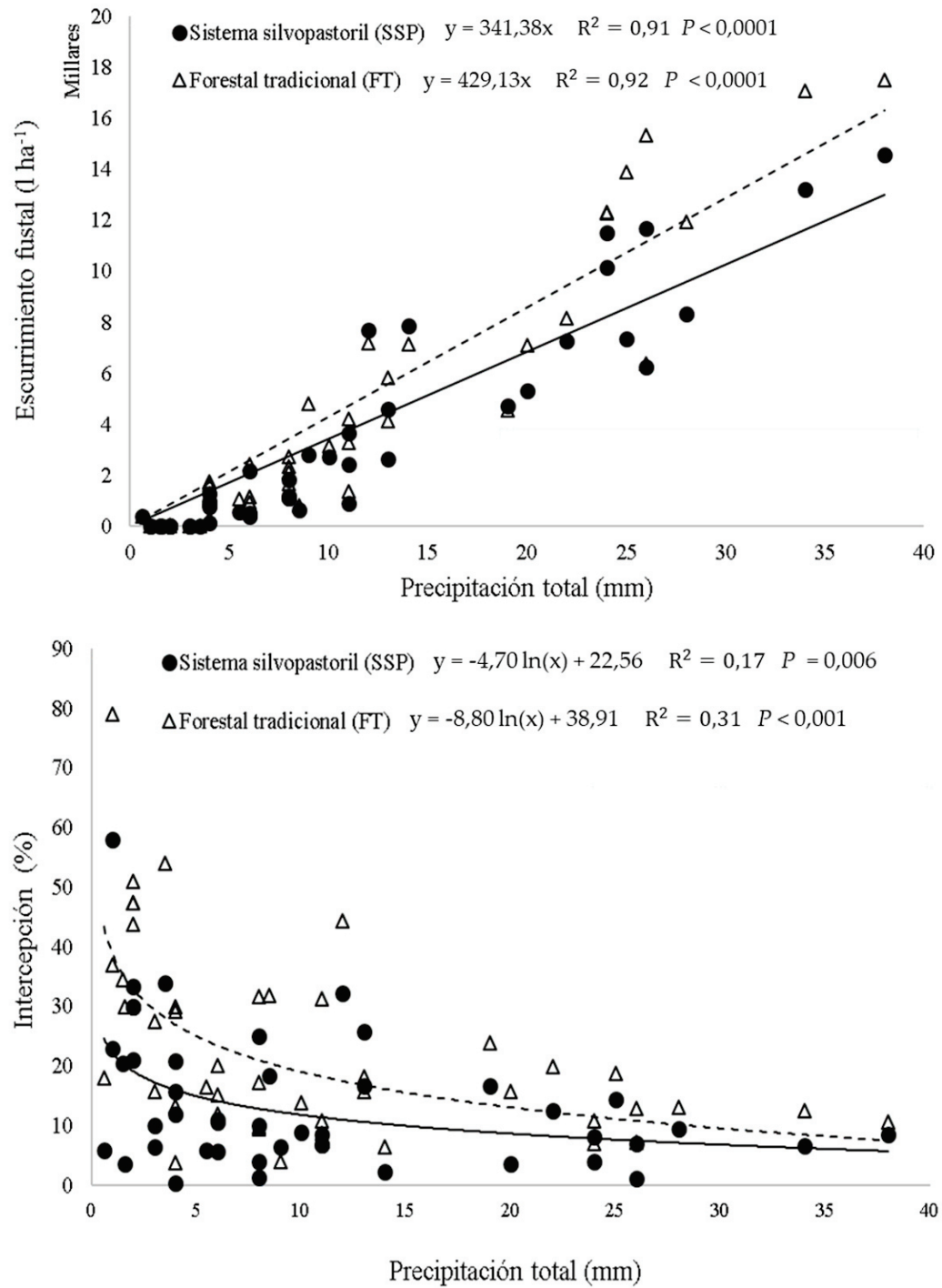

Figura 4. Escurrimiento fustal registrado en el manejo silvopastoril (-) y en el forestal tradicional (---).

Figure 4. Stemflow in silvopastoral system (-) and forest system (---). se encontraba saturado y con poca capacidad de seguir almacenando agua.

\section{Contenido de humedad del suelo}

A lo largo del año, el contenido de humedad del suelo fue significativamente mayor en el sistema silvopastoril $(24.2 \pm 2.5 \%)$ que en el manejo forestal tradicional $(20.9 \pm 2.1 \%)(t=-$ 5.22; $P<0.01)$, aunque en la estación seca la diferencia tendió a disminuir (Figura 6).

\section{Percolación}

A lo largo del estudio, las pérdidas por percolación resultaron de $0.33 \pm 0.12 \%$ para el sistema silvopastoril y de $0.11 \pm 0.08 \%$ para el forestal tradicional, sin presentar diferencias significativas $(P=0.15)$.

\section{Evapotranspiración}

A lo largo del período analizado, la evapotranspiración fue $861.3 \mathrm{~mm}$ en el silvopastoril y $807.4 \mathrm{~mm}$ en el forestal tradicional. Esto representa el 78.2\% y el 73.3\% de la precipitación incidente $(1101.2 \mathrm{~mm})$, respectivamente, sin presentar diferencias significativas $(\mathrm{t}=0.71 ; P>0.05)$.

\section{Producción de los pastos}

La producción de pastos fue 230.76 297.57 $\mathrm{kg}$ MS.ha- ${ }^{-1}$. año ${ }^{-1}$ para el sistema silvopastoril

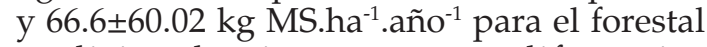
tradicional, sin presentar diferencias significativas $(P=0.29)$. Las especies dominantes en ambas parcelas fueron Holcus lanatus L., Dactylis glomerata L., Trifolium repens L. y Bromus spp. 


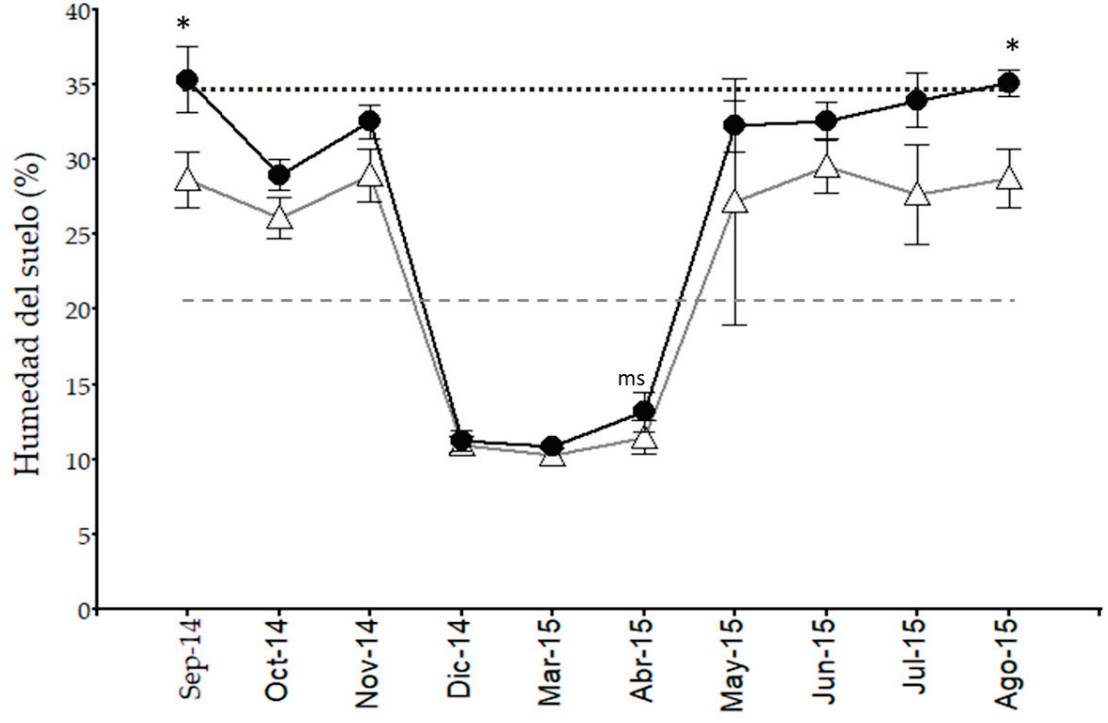

Mes
Figura 6. Valores medios y error estándar para el contenido de humedad del suelo a $50 \mathrm{~cm}$, en el sistema silvopastoril $(\bullet)$ y en el forestal tradicional $(\Delta)$. CC: capacidad de campo, PMP: punto de marchitez permanente. * Diferencias significativas; ms: $\mathrm{m}$ a r gin a lm en te significativo.

Figure 6. Mean values and standard error for soil moisture content under silvopastoral system $(\bullet)$ and forest system $(\Delta)$. CC: field capacity, PMP: permanent wilting point. * Significant differences; ms: marginally significant.
- Sistema silvopastoril $\longrightarrow$ Forestal tradicional

\section{Balance hidrológico}

La descomposición de los distintos componentes del balance del agua muestra que en el sistema silvopastoril (Figura 7A) el ingreso de agua al suelo $\left(\mathrm{P}_{\mathrm{d}}+\mathrm{E}_{\mathrm{f}}\right)$ es mayor que en el sistema forestal (Figura 7B). Esto determina mayores valores de humedad de suelo. Sin embargo, la evapotranspiración no presentó diferencias significativas entre tratamientos $(P>0.05)$.

\section{DISCUSIÓN}

Los valores de los componentes de la precipitación hallados en este trabajo

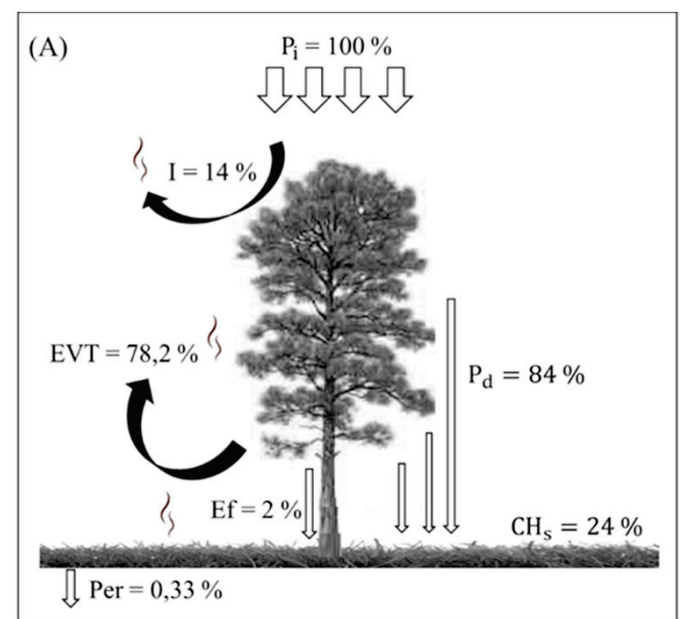

coinciden con otros estudios realizados en plantaciones similares en Chile y la Argentina. Los valores de precipitación directa fueron mayores en el manejo silvopastoril que en el forestal tradicional. En una plantación de Pinus radiata en Chile se registraron valores de precipitación directa de $74 \%$ para un rodal de 26 años con 733 árboles/ha y de $80 \%$ para un sistema silvopastoril de 9 años con 443 árboles/ha (Huber et al. 1985), con una tendencia similar al presente estudio. Por otra parte, en plantaciones de Pinus ponderosa en cercanías a Esquel, provincia de Chubut, y con más de 1000 árboles/ha, la precipitación directa media fue $62 \%$ del total

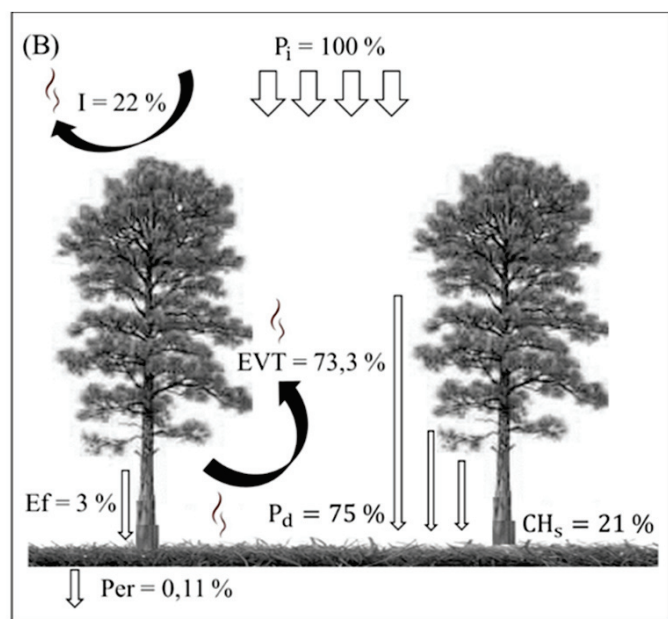

Figura 7. Componentes del ciclo hidrológico. (A): sistema silvopastoril con 325 árboles/ha. (B): sistema forestal tradicional con 622 árboles/ha. $\mathrm{P}_{\mathrm{i}}$ : precipitación incidente. $\mathrm{P}_{\mathrm{d}}$ : precipitación directa. $\mathrm{E}_{\mathrm{f}}$ : escurrimiento fustal. I: intercepción. $\mathrm{CH}_{\mathrm{s}}$ : contenido de humedad del suelo. Per: percolación. EVT: evapotranspiración.

Figure 7. Components of the hydrological cycle. (A): silvopastoral system. (B): forest system. $P_{i}$ : gross precipitation. $P_{d}$ : throughfall. $\mathrm{E}_{\mathrm{f}}$ : stemflow. I: interception. $\mathrm{CH}_{\mathrm{s}}$ : soil moisture content. Per: percolation. EVT: evapotranspiration. 
de las precipitaciones (Buduba 2006). Otros trabajos, también con plantaciones de Pinus ponderosa, la estimaron entre 78 y $82 \%$ (Licata et al. 2011), y $95 \%$ de precipitación directa (Gyenge et al. 2002).

Con respecto a la precipitación incidente, el escurrimiento fustal presentó valores bajos tanto en el manejo silvopastoril como en el forestal tradicional. En una plantación de 26 años de Pinus radiata en Chile, con una densidad de 733 árboles/ha, evidenciaron valores de escurrimiento fustal de entre 5.7 y $14.8 \%$ (Huber and Oyarzún 1983). Buduba (2006) obtuvo $2.9 \%$ de escurrimiento fustal en plantaciones de Pinus ponderosa con una densidad >1000 árboles/ha. Diferentes autores encontraron valores muy similares; en abetos y pinos se registraron valores entre 1 y $2 \%$ de escurrimiento fustal (Aussenac 1968); en Pinus pinea L. se obtuvieron registros de entre 2 y 3\% (Rapp and Ibrahim 1978). Por el contrario, en Picea sitchensis (Bong. Carr.) se encontraron valores cercanos a $27 \%$ (Ford and Deans 1978). Estas variaciones se deben principalmente a las características de las especies, a la estructura, la edad y el manejo de los bosques (Santa et al. 1989). El mayor escurrimiento fustal registrado en la parcela forestal tradicional de este estudio puede estar influenciado por el mayor número de árboles incluidos en la parcela, ya que se asume que todos ellos canalizaron el agua de igual manera que los ejemplares dominantes seleccionados.

Respecto al manejo forestal tradicional, la intercepción fue menor en el sistema silvopastoril. Esto se debe a la menor cobertura arbórea que presentó el sistema silvopastoril con respecto al forestal tradicional (Tabla 1). Otros autores, en plantaciones de Pinus radiata, registraron un máximo de $53 \%$ de pérdida por intercepción, con un promedio del 10\% para precipitaciones muy intensas (Huber and Oyarzún 1983). En plantaciones de Pinus ponderosa con 1000 árboles/ha se registró un promedio de $35.4 \%$ de intercepción (Buduba 2006). También, en P. ponderosa en Patagonia se encontraron valores de intercepción similares al presente estudio: entre 17 y $22 \%$ (Licata et al. 2011) y 5\% (Gyenge et al. 2002). Aussenac (1968) remarca que la pérdida por intercepción es muy importante en coníferas, y que se encuentran valores altos en distintas especies, como 30\% en Pinus sylvestris L., $34 \%$ en Picea abies (L.) Karst. y hasta $42 \%$ en Abies grandis (Dougl.) Lindl. Mientras tanto, en especies caducifolias, la intercepción es más baja debido a su morfología (Santa et al. 1989).

El mayor ingreso de agua al suelo en el manejo silvopastoril, asociado a una menor intercepción de las lluvias, se reflejó en el mayor contenido de humedad del suelo. Si bien esto implica una mayor disponibilidad de agua para las especies forrajeras que componen el sistema, este efecto parece atenuarse en la época seca (Figura 6). Asimismo, Gyenge et al. (2002) encontraron que el contenido de agua en los pastos es mayor bajo manejos silvopastoriles en Patagonia, cuando se compara con sitios sin árboles, antes y después del mes más seco (enero). Si bien el contenido hídrico en el verano estuvo por debajo del punto de marchitez permanente en ambos manejos, no necesariamente indica un déficit hídrico, ya que se demostró que $P$. radiata es capaz de mantener turgencia en las acículas, aun en condiciones de suelo muy seco (Johnston 1964). Así, si bien los valores de percolación resultaron muy bajos, el muestreo de humedad de suelo a los 50 $\mathrm{cm}$ abarca la profundidad explorada por las raíces de los pastos y sólo parcialmente la profundidad explorada por el componente arbóreo (Bassman 1987; Schulze et al. 1996; Gyenge et al. 2002). En el área de estudio, la profundidad efectiva de los suelos varía entre 70 y $100 \mathrm{~cm}$, según datos de calicatas (Vallejos et al. 2009).

A lo largo del período de estudio, la percolación, estimada como la diferencia entre CC y la humedad del suelo a los 50 $\mathrm{cm}$, resultó mínima en ambos tratamientos. Estudios realizados en Chile indican que el factor percolación en plantaciones $P$. radiata puede ser muy variable, con valores entre 0 y $54 \%$ en función de la edad, el manejo y la condición de sitio (Huber et al. 2007).

La evapotranspiración no presentó diferencias significativas entre los tratamientos (silvopastoril y forestal tradicional). La evapotranspiración estimada representa en parte el agua utilizada por los pastos y una porción no cuantificada utilizada por parte del componente arbóreo. Dicho esto, se está subestimando la evapotranspiración de la parte arbórea y, probablemente, más aun en el manejo forestal tradicional, que posee casi el doble de árboles que el sistema silvopastoril, aunque los valores de IAF resultaron semejantes. Autores como Huber et al. $(1985,2007)$ encontraron valores de evapotranspiración de entre 31 y $62 \%$ para 
distintos manejos en Pinus radiata. Cabe destacar que la escorrentía superficial no fue considerada en el análisis por tratarse de sitios planos (menores a $2 \%$ de pendiente) (Huber et al. 1985).

Los resultados de este estudio muestran que la densidad de plantación de coníferas exóticas afectó sólo algunos de los componentes del balance hídrico, lo que permite no rechazar la hipótesis planteada. En el sistema silvopastoril ingresó más agua por precipitación directa, lo que se tradujo en mayor contenido de humedad del suelo y una tendencia a incrementar la producción de pastos. Sin embargo, la producción de pastos en ambos sistemas resultó baja, lo que indica que los dos sistemas están limitados por radiación. A pesar de las intervenciones realizadas en el sistema silvopastoril, que redujeron en 50\% la densidad arbórea, ambos tratamientos presentaron valores de IAF similares. Los resultados muestran que el manejo silvopastoril, con $70 \%$ de cobertura del dosel, continúa limitando la producción pastoril. Para optimizar el componente forrajero del sistema silvopastoril con bosque implantado es necesario hacer un raleo que baje la cobertura hasta valores de entre 30 y $50 \%$ a fin de mejorar la producción de las pasturas (Varela et al. 2015).

Los resultados de este estudio contribuyen a un mejor entendimiento del manejo de las plantaciones de la Región Patagónica, donde existen aproximadamente 80000 ha de bosque implantadas con coníferas exóticas del género Pinus (Loguercio and Deccechis 2006). Es de suma importancia generar información de las interacciones ecológicas y ambientales en los sistemas silvopastoriles, a fin de maximizar los beneficios tanto del componente forestal como del pastoril y del ganadero.

Agradecimientos. Agradecemos al Campo Experimental INTA EEAf de Esquel-Chubut por brindarnos el uso de los sitios de muestreo y de los materiales necesarios para realizar este presente estudio. Además, agradecer al Centro de Estudios Ambientales Integrados (CEAI) perteneciente a la Facultad de Ingeniería de la Universidad Nacional de la Patagonia San Juan Bosco (FI-UNPSJB), que nos permitió realizar todas las determinaciones analíticas en su laboratorio y demás trabajos de gabinete.

\section{REFERENCIAS}

Alinari, M. J.; A. R. von Müller, and D. Renison. 2015. The contribution of fire damage to restricting high mountain Polylepis australis forests to ravines: Insights from an un-replicated comparison. Ecología Austral 25:11-18.

Allen, G. R., L. Pereira, D. Raes, and M. Smith. 2006. Introducción a la evapotranspiración. En: Evapotranspiración del cultivo - Estudio FAO Riego y drenaje 56. Organización de las Naciones Unidas para la Agricultura y la Alimentación. Roma. Cap 1: 1-13. ISBN 92-5-304219-2.

Andrade, L. 2002. Territorio y ganadería en la Patagonia Argentina: desertificación y rentabilidad en la Meseta Central de Santa Cruz. Economía, Sociedad y Territorio 3(12). https:/ /doi.org/10.22136/est002002341.

Aussenac, G. 1968. Interception des précipitations par le couvert forestier. Annales des Sciences Forestieres 25(3):35156. https://doi.org/10.1051/forest/19680302.

Bahamonde, H. A., P. L. Peri, G. Martínez Pastur, and M. V. Lencinas. 2009. Variaciones microclimáticas en bosques primarios y bajo uso silvopastoril de Nothofagus antarctica en dos clases de sitio en Patagonia Sur. En Primer congreso silvopastoril, Posadas, Misiones, Argentina. Pp. 289-296.

Barradas, V. L. 1983. Capacidad de captación de agua a partir de la niebla en Pinus montezumae Lambert, de la región de las Grandes Montañas del Estado de Veracruz. Biótica 8(4):427-431.

Bassman, J. H. 1987. Photosynthesis and water relations of Ponderosa Pine. Pp. 45-58 in Baumgartner, D. M. and J. E. Lotar (eds.). Ponderosa Pine. The species and its management. Symposium proceedings, Sept 29-Oct 1. Spokane, Washington, USA.

Branson, F. A., G. F. Gifford, and K. G. Renard. 1981. Rangeland hydrology. Society for Range Management. Kendall / Hunt Publishing Company. USA. Pp. 340.

Buduba, C. G. 2006. Modificaciones en el pH y contenido de materia orgánica en suelos del ecotono estepa / bosque andino patagónico por implantación de pino ponderosa. Tesis doctoral. Facultad de Agronomía, Universidad de Buenos Aires. Pp. 176.

Carranza, C., and M. Ledesma. 2009. Bases para el manejo de sistemas silvopastoriles. In Actas XIII Congreso Forestal Mundial, Buenos Aires, Argentina. Pp. 9.

Crockford, R., and D. Richardson. 2000. Partitioning of rainfall into throughfall, stemflow and interception: effect of forest type, ground cover and climate. Hydrological Processes 14(16-17):2903-2920. https:/ /doi.org/10.1002/10991085(200011/12)14:16/17\%3C2903::AID-HYP126\%3E3.0.CO;2-6.

Ford, E., and J. Deans. 1978. The effects of canopy structure on stemflow, thoughfall and interception loss in a young sitka spruce plantation. Journal of Applied Ecology 15:905-917. https://doi.org/10.2307/2402786.

Gyenge, J. E., M. E. Fernández, J. A. Licata, M. N. Weigandt, B. J. Bond, and T. Schlichter. 2011. Uso del agua y productividad de los bosques nativos e implantados en el NO de la Patagonia: aproximaciones desde la ecohidrología y la ecofisiología.

Gyenge, J. E., M. E. Fernández, G. Dalla Salda, and T. M. Schlichter. 2002. Silvopastoral systems in Northwestern Patagonia II: water balance and water potential in a stand of Pinus ponderosa and native grassland. Agroforestry Systems 55(1):47-55. https:/ / doi.org/10.1023/A:1020269432671. 
Gyenge, J., M. E. Fernández, and T. Schlichter. 2009. Effects on site water balance of conversion from native mixed forest to Douglas-fir plantation in NW Patagonia. New Forests 38(1):67-80. https:/ /doi.org/10.1007/s11056-009-9132-0.

Hargrove, W. W., and J. Pickering. 1992. Pseudoreplication: a sine qua non for regional ecology. Landscape Ecology 6(4):251-258. https://doi.org/10.1007/BF00129703.

Huber, A., A. Iroumé, and J. Bathurst. 2007. Effect of Pinus radiata plantations on water balance in Chile. Hydrological Processes 22(1):142-148. https:// doi.org/10.1002/hyp.6582.

Huber, A., and C. Oyarzún. 1983. Precipitación neta e intercepción en un bosque adulto de Pinus radiata (D. Don). Bosque 5(1):13-20. https:/ / doi.org/10.4206/bosque.1983.v5n1-02.

Huber, A., and C. Oyarzún. 1984. Factores reguladores de la intercepción en un bosque adulto de Pinus radiata (D. Don). Bosque 5(2):59-64. https:// doi.org/10.4206/bosque.1986.v7n2-01. https://doi.org/10.4206/bosque.1984.v5n2-02.

Huber, A., and D. López. 1993. Cambio en el balance hídrico provocado por la tala rasa de un rodal adulto de Pinus radiata (D. Don), Valdivia Chile. Bosque 14(2):11-18. https:/ /doi.org/10.4206/bosque.1993.v14n2-02.

Huber, A., and R. Trecamán. 2004. Eficiencia del uso del agua en plantaciones de Pinus radiata en Chile. Bosque (Valdivia) 25(3):33-43. https://doi.org/10.4067/S0717-92002004000300004.

Huber, A., C. Oyarzún, and A. Ellies. 1985. Balance hídrico en tres plantaciones de Pinus radiata y una pradera. II: Humedad del suelo y evapotranspiración. Bosque 6(2):74-82. https://doi.org/10.4206/bosque.1985.v6n2-02.

Huber, A., P. Barriga, and R. Trecaman. 1998. Efecto de la densidad de plantaciones de Eucalyptus nitens sobre el balance hídrico en la zona de Collipulli, IX Región (Chile). Bosque 19(1):61-69. https:/ / doi.org/10.4206/bosque.1998.v19n107.

Jaramillo-Robledo, A., and B. Cháves-Córdoba. 1999. Aspectos hidrológicos en un bosque y en plantaciones de café (Coffea arabica L.) al sol y bajo sombra. Cenicafé 50(2):97-105.

Jobbágy, E. G., M. Vasallo, K. A. Farley, G. Piñeiro, M. F. Garbulsky, M. D. Nosetto, and J. M. Paruelo. 2006. Forestación en pastizales: hacia una visión integral de sus oportunidades y costos ecológicos. Agrociencia 10(2):109-124.

Johnston, R. D. 1964. Water relations of Pinus radiata under plantation conditions. Australian Journal of Botany 12(2): 111-124. https://doi.org/10.1071/BT9640111.

Kimmins, J. P. 1997. Forest ecology. A foundation for sustainable management. Prentice-Hall, Inc. U.S.A. Pp. 596.

La Manna, L., C. Buduba, and J. Irisarri. 2008. Los Suelos del Valle 16 de Octubre, Provincia del Chubut. Patagonia Forestal 14(2):13-16.

Licata, J. A., T. G. Pypker, M. Weigandt, M. H. Unsworth, J. E. Gyenge, M. E. Fernández, T. M. Schlichter, and B. J. Bond. 2011. Decreased rainfall interception balances increased transpiration in exotic ponderosa plantations compared with native cypress stands in Patagonia, Argentina. Ecohydrology 4:283-293. https://doi.org/10.1002/eco.125.

Loguercio, G., and F. Deccechis. 2006. Forestaciones en la Patagonia Andina: potencial y desarrollo alcanzado. Patagonia Forestal 12(1):4-6.

López Ritas, J., and J. López Melida. 1990. El diagnóstico de Suelos y Plantas (métodos de campo y laboratorio). Ediciones Mundi-Prensa, Madrid.

Mahecha, L. 2002. El silvopastoreo: una alternativa de producción que disminuye el impacto ambiental de la ganadería bovina. Revista Colombiana de Ciencias Pecuarias 15(2):226-231.

Martínez Pastur, G. J., J. M. Cellini, M. V. Lencinas, R. Soler Esteban, H. A. Bahamonde, and P. L. Peri. 2015. Monitoreo de copas empleando fotos hemisféricas en raleos de Nothofagus antarctica en Tierra del Fuego. En Congreso Nacional de Sistemas Silvopastoriles. 3. Congreso Internacional de Sistemas Agroforestales. 8. 2015 05 07-09, 7, 8 y 9 de mayo de 2015. Iguazú, Misiones. Argentina.

Mussat, E., M. Fariña, and P. Laclau. 2013. El modelo forestal neuquino y el desarrollo rural del departamento Minas. Realidad Económica 276:129-154.

Nair, P., B. Mohan Kumar, and V. D. Nair. 2009. Agroforestry as a strategy for carbon sequestration. Journal of plant nutrition and soil science 172(1):10-23. https:/ / doi.org/10.1002/jpln.200800030.

Oyarzún, C., A. Huber, and S. Vásquez. 1985. Balance hídrico en tres plantaciones de Pinus radiata. I: Redistribución de las precipitaciones. Bosque 6:3-14. https://doi.org/10.4206/bosque.1985.v6n1-01.

Pérez, E., M. Soca, L. Díaz, and M. Corzo. 2008. Comportamiento etológico de bovinos en sistemas silvopastoriles en Chiapas, México. Pastos y Forrajes 31(2):161-171.

Rapp, M., and M. Ibrahim. 1978. Egouttement, écoulement et interception des précipitations par un peuplement de Pinus pinea L. Oecologia Plantarum 13:321-330.

Santa, R., J. Gallardo, C. San Miguel, and A. Moyano. 1989. Intercepción, pluviolavado y escorrentía cortical en una plantación de Pinus sylvestris de la Cuenca de Candelario (Centro-Oeste de España). Bosque 10(1):19-27. https:// doi.org/10.4206/bosque.1989.v10n1n2-03.

Schlichter, T., and P. Laclau. 1998. Ecotono estepa-bosque y plantaciones forestales en la Patagonia norte. Ecología Austral 8(2):285-296.

Schulze, E. D., H. A. Mooney, O. E. Sala, E. Jobbágy, N. Buchmann, G. Bauer, and J. R. Ehleringer. 1996. Rooting depth, water availability, and vegetation cover along an aridity gradient in Patagonia. Oecologia 108(3):503-511. https: //doi.org/10.1007/BF00333727.

Soil Survey Staff. 2010. Claves para la Taxonomía de Suelos, 11th ed. USDA-Natural Resources Conservation Service, Washington, D.C.

Vallejos, N., L. La Manna, C. Buduba, and D. Aerrechea. 2009. Base de datos de suelos de la Región Patagónica Andina. Patagonia Forestal 15(2):18-19.

Varela, S., G. Caballé, J. P. Diez, and M. Godoy. 2015. Efecto de la cobertura arbórea sobre la instalación de especies alternativas al Pino ponderosa en sistemas silvopastoriles del NO Patagónico. Sustentable. I. Peri, Pablo L., comp. II. Título 634.0, 98.

Zuazo, V. D., A. Martínez-Raya, and J. A. Ruiz. 2002. Control de la erosión en los taludes de bancales, en terrenos con fuertes pendientes. Edafología 9(1):1-10. 